\title{
INFLUÊNCIA DO AMBIENTE NO RENDIMENTO E NA QUALIDADE DE GRÃOS DE GENÓTIPOS DE TRIGO COM IRRIGAÇÃO POR ASPERSÃO NO ESTADO DE SÃO PAULO ${ }^{(1)}$
}

\author{
JOÃO CARLOS FELICIO ${ }^{(2)}$; CARLOS EDUARDO DE OLIVEIRA \\ CAMARGO $^{(2,8)}$; ROGÉRIO GERMANI ${ }^{(3)} ;$ PAULO BOLLER GALLO $^{(4)}$; \\ JOSÉ CARLOS VILA NOVA ALVES PEREIRA ${ }^{(5)}$; NELSON BORTOLETTO ${ }^{(6)}$; \\ ARMANDO PETTINELLI JUNIOR ${ }^{(7)}$ \\ RESUMO
}

Avaliaram-se a influência do ambiente em 20 genótipos de trigo, no rendimento e qualidade de grãos, a adaptabilidade e a reação a doenças, em regiões onde a irrigação se faz necessária para permitir o bom desempenho agronômico de um genótipo. Instalaram-se os experimentos em Tatuí, Votuporanga, Ribeirão Preto e Mococa, Estado de São Paulo, no período de 1995-98. Avaliaram-se, também, a tolerância dos genótipos ao alumínio tóxico em solução nutritiva e a qualidade industrial para panificação. Com bom rendimento de grãos destacaram-se: em Tatuí, os genótipos IAC 351, IAC 335, IAC 289 e Mochis; em Votuporanga, IAC 289, TUI“S” e SERI*3/BUC; em Ribeirão Preto e Mococa, o IAC 370. No conceito de genótipo ideal, o IAC 370 apresentou alta capacidade produtiva, foi responsivo à melhoria do ambiente e sensível às condições desfavoráveis do ambiente. Entre as doenças, a ferrugem-da-folha foi a de abrangência generalizada com maior incidência em Tatuí. Anahuac, IAC 287, CAL/CHKW/ /VEE"S" e IAC 370 foram os mais suscetíveis ao oídio. Os genótipos Anahuac, IAC 287, JCAM/ /EMU“S" /YACO"S", PFAU e IAC 339 foram os mais sensíveis à toxicidade por $\mathrm{Al}^{3+}$. Os genótipos Anahuac, IAC 24, IAC 287, IAC 289, IAC 334, PFAU, TUI“S”, IAC 339, IAC 370 e IAC 351 apresentaram características de farinha com bom potencial para panificação.

Palavras-chave: trigo, rendimento de grãos, doenças, tolerância ao $\mathrm{Al}^{3+}$, adaptação ampla, característica tecnológica da farinha.

\section{ABSTRACT \\ ENVIRONMENTAL INFLUENCE ON GRAIN YIELD AND GRAIN QUALITY OF WHEAT GENOTYPES WITH SPRINKLER IRRIGATION IN THE STATE OF SÃO PAULO, BRAZIL}

Environmental influence was evaluated in 20 wheat genotypes in relation to grain yield and quality, adaptability and disease reaction in regions where irrigation is necessary for good agronomical performance. The experiments were carried out at Tatuí, Votuporanga, Ribeirão Preto, and Mococa in

(1) Trabalho realizado com apoio da FAPESP. Recebido para publicação em 22 de novembro de 2000 e aceito em 19 de julho de 2001.

$\left({ }^{2}\right)$ Centro de Plantas Graníferas, Instituto Agronômico (IAC), Caixa Postal 28, 13001-970 Campinas (SP). E-mail: jfelicio@cec.ia.br

(3) Embrapa, Centro Nacional de Pesquisa de Tecnologia Agroindustrial de Alimento (CTAA), 23020-470 Rio de Janeiro (RJ).

$\left({ }^{4}\right)$ Estação Experimental de Agronomia de Mococa, IAC, Caixa Postal 58, 13730-970 Mococa (SP).

(5) Núcleo de Agronomia da Alta Mogiana, IAC, Caixa Postal 271, 14001-970 Ribeirão Preto (SP).

(6) Núcleo de Agronomia do Noroeste, IAC, Caixa Postal 401, 15500-000 Votuporanga (SP).

(7) Estação Experimental de Agronomia de Tatuí, IAC, Caixa Postal 33, 18270-000 Tatuí (SP).

( ${ }^{8}$ Com bolsa de produtividade em pesquisa do CNPq. 
the State of São Paulo, Brazil, during 1995-98. Genotypes were also evaluated for tolerance to aluminum toxicity in nutrient solutions and for industrial quality for bread production. The genotypes IAC 351, IAC 335, IAC 289 and Mochis presented high grain yield in Tatuí; IAC 289, TUI"S" and SERI*3/BUC in Votuporanga; and IAC 370 in Ribeirão Preto and Mococa. Under the ideal genotype concept the IAC 370 showed high grain yield capacity, was responsive to the environment improvement and sensitive to the unfavorable environment conditions. Among the diseases, leaf rust presented general occurrence, showing higher incidence in Tatuí. Anahuac, IAC 287, CAL/CHKW / /VEE"S" and IAC 370 were the most susceptible to powdery mildew. The genotypes Anahuac, IAC 287, JCAM//EMU"S"/YACO"S", PFAU and IAC 339 were the most sensitive to aluminum toxicity. Anahuac, IAC 24, IAC 287, IAC 289, IAC 334, PFAU, TUI"S", IAC 339, IAC 370 and IAC 351 exhibited flour characteristics with good potential for bread production.

Key words: wheat, grain yield, diseases, aluminum tolerance, high adaptation, flour technological characteristics.

\section{INTRODUÇÃO}

O cerrado é um sistema solo-planta-atmosfera, cuja característica principal é a baixa fertilidade e alta acidez de seus solos; $25 \%$ do território nacional constitui-se de cerrado. Práticas racionais de agricultura, como a correção do $\mathrm{pH}$ do solo, fertilização adequada e um correto manejo de água, podem levar a produtividades altamente compensadoras (REICHARDT, 1985). GOEDERT et al. (1980) definem sub-regiões de cerrado em Mato Grosso, Sul de Goiás e Norte de São Paulo, as quais apresentam influência climática austral continental, sendo regiões mais frias e secas que as demais regiões de cerrado do Brasil. Segundo Malavolta e Kliemann (1985), solos de cerrado caracterizam-se por alta acidez, acompanhada por altos teores de alumínio trocável.

Segundo AzEvEDo et al. (1983), o regime de chuvas das regiões de cerrado é constituído por duas estações definidas e distintas. Uma é o período chuvoso compreendido entre outubro e março e com $80 \%$ a $90 \%$ da precipitação anual, concentrando maior atividade agrícola nesse período e a outra, de abril a setembro, denominada estação seca, com baixa atividade agrícola.

A cultura do trigo é uma opção para o período março-setembro em sucessão às culturas tradicionalmente semeadas no verão. A triticultura paulista, até o início da década de 80, instalava-se no Vale do Paranapanema e no Sul do Estado, onde o trigo era semeado em sucessão à soja, e o cultivo feito em condições de sequeiro. A partir de 1982, com o estabelecimento da cultura irrigada por aspersão, foi possível o cultivo do trigo em todo o Estado, pois a deficiência hídrica, característica do inverno paulista, pôde ser facilmente eliminada por essa prática.

A expressão do potencial de rendimento de um genótipo em uma região depende de fatores genéticos e ambientais, especialmente o fotoperíodo, a temperatura e a radiação solar. Eventos climáticos catastróficos, como geadas, granizos, excesso ou deficiência de precipitações pluviais, têm também efeitos importantes sobre o potencial de rendimento (WALL, 1997).

FeLICIO et al. (1996), avaliando cultivares de trigo em condições de irrigação por aspersão no Estado de São Paulo, concluíram que os genótipos de trigo IAC 289, IAC 335, IAC 338, IAC 286 e IAC 60 apresentaram bom rendimento de grãos, enquanto os genótipos IAC 24, IAC 315, IAC 334, TUI"S" e IAC 339 se destacaram pela qualidade de sua farinha.

Com o objetivo de avaliar 20 genótipos de trigo, quanto ao rendimento e à qualidade de grãos, adaptabilidade, estabilidade e reação a doenças, foram instalados experimentos com irrigação por aspersão, em diferentes ambientes (locais) no Estado de São Paulo, no período 1995-98; o comportamento desses genótipos, em solução nutritiva contendo níveis crescentes de alumínio trocável, também foi avaliado.

\section{MATERIAL E MÉTODOS}

Visando avaliar o comportamento agronômico e a qualidade industrial para a panificação de genótipos de trigo, nas condições de irrigação por aspersão, instalaram-se no período 1995-98, experimentos nas seguintes localidades tritícolas paulistas: zona $\mathbf{D}$, Tatuí; zona F, Votuporanga; zona G, Ribeirão Preto, e zona $\mathbf{H}$, Mococa. Para a instalação dos experimentos seguiram-se as recomendações da Comissão Técnica de Trigo, da Secretaria de Agricultura e Abastecimento do Estado de São Paulo (CAMPINAS, 1999).

Os experimentos foram delineados em blocos casualizados, compostos de 20 genótipos com quatro repetições por local. Cada parcela foi constituída por seis linhas de $3 \mathrm{~m}$ de comprimento, com espaçamento de $0,20 \mathrm{~m}$ entre si e separação lateral de $0,60 \mathrm{~m}$ entre as parcelas. Procedeu-se à semeadura com 80 sementes por metro de sulco, realizando-se a colheita na área total das parcelas.

A adubação mineral foi feita a lanço antes da semeadura e, posteriormente, incorporada ao solo. Definiu-se a quantidade de fertilizantes a ser aplicada 
nos diferentes locais, considerando-se as recomendações de adubação e calagem para o Estado de São Paulo (RAIJ et al., 1996).

Foram avaliados, quanto ao rendimento de grãos, resistência a doenças e qualidade tecnológica, os seguintes genótipos: Anahuac, IAC 24, IAC 60, IAC 286, IAC 287, IAC 289, JCAM / / EMU “S" /YACO "S", IAC 315, IAC 334, IAC 335, IAC 352, Mochis, CAL/CHKW//VEE“S”, PFAU, TUI“S”, IAC 339, BAU/SERI, SERI*3/BUC, IAC 370 e IAC 351.

A ferrugem-da-folha (Puccinia triticina Rob. ex Desm. F. sp. tritici Erickss) foi avaliada após o florescimento das plantas (estádio de crescimento 11.2 escala de Large, 1954) por meio da escala modificada de Cobb, conforme Schramm et al. (1974). Avaliou-se, também, a porcentagem de área foliar infectada por Bipolaris sorokiniana, Drechslera tritici repentis e Blumeria graminis, respectivamente, mancha-marrom, mancha-bronzeada-da-folha e oídio.

Nos experimentos, adotou-se o método de irrigação proposto por SILVA et al. (1984): uma irrigação inicial após a semeadura, de 40 a $60 \mathrm{~mm}$, a fim de umedecer o perfil do solo, quando foram instalados os tensiômetros em diversos pontos, à profundidade de $12 \mathrm{~cm}$; realizaram-se irrigações complementares quando a média das leituras dos tensiômetros indicava -0,06 Mpa; determinou-se a lâmina líquida aplicada pela evaporação acumulada, medida no tanque classe A, entre os intervalos das irrigações.

Avaliou-se o rendimento de grãos, em gramas, pesando-se a produção total de cada parcela, a qual foi transformada para quilograma por hectare.

Para os experimentos, realizaram-se análises da variância conjunta por zona tritícola, visando avaliar o efeito de anos, genótipos e a interação anos x genótipos. Para comparação das médias, utilizou-se o teste de Duncan, de acordo com Gomes (1990).

Determinaram-se a estabilidade e a adaptabilidade dos genótipos por região, bem como em todas as regiões em conjunto, pelo método proposto por EBERHART e RusSel (1966), com base nos coeficientes de cada genótipo em relação ao índice ambiental. Considerou-se, como genótipo ideal, aquele com alto rendimento médio de grãos, coeficiente de regressão igual a $1,0(b=1)$ e desvio de regressão igual a zero $\left(\mathrm{S}^{2} \mathrm{~d}=0\right)$.

A avaliação da qualidade tecnológica dos genótipos foi realizada somente nos experimentos da zona G (Ribeirão Preto), durante o biênio 1996-97. As produções de grãos das parcelas das quatro repetições de cada experimento (ano) foram reunidas para obter quantidade suficiente de grãos para as determinações a seguir relacionadas.
Teor de água dos grãos: determinada pelo método n. ${ }^{\circ}$ 44-15A da American Association of Cereal Chemists - AACC (1995).

Peso hectolítrico: conforme procedimento descrito na Instrução Normativa n. ${ }^{\circ} 1$, do Ministério da Agricultura e do Abastecimento (BRASIL, 1999), utilizando-se a balança "Dalle Molle".

Rendimento de extração de farinha (moagem): empregando-se o moinho experimental "Brabender Quadrumat Senior", em amostras de $2 \mathrm{~kg}$ de grãos, com base no método da AACC n. ${ }^{\circ}$ 26-10A (AACC, 1995). Os grãos tiveram seu teor de água ajustado para 15\%, 16-24 horas antes da moagem. A extração de farinha representa a porcentagem da obtida em relação ao total do material resultante da moagem.

Proteína da farinha: determinada pelo método 920.87, da Association of Official Analytical Chemists - AOAC (1997), expressa em base seca.

Número de queda (Falling number): determinado pelo método $\mathrm{n} .{ }^{\text {. }}$ 56-81B da AACC (AACC, 1995), em grãos triturados em moinho Perten 3100 (Perten Instruments, Huddinge, Suécia), com peneira de $0,8 \mathrm{~mm}$.

Propriedades de mistura da massa: determinadas no farinógrafo "Brabender", pelo sistema farinhaágua, de acordo com o método n. ${ }^{\circ}$ 54-21 da AACC (AACC, 1995). Misturou-se $50 \mathrm{~g}$ de farinha (na base de $14 \%$ de umidade) com água em quantidade suficiente para centralizar a curva na linha de 500 unidades farinográficas (U.F.). Essa quantidade de água, expressa em porcentagem em relação à farinha, é referida como "absorção". O tempo de desenvolvimento da massa (TDM) é o tempo (em minutos) necessário para alcançar a consistência máxima; estabilidade (EST) é o tempo (em minutos) em que a curva permanece na linha de 500 U.F.; índice de tolerância à mistura (ITM) é a diferença (em U.F.) medida no topo da curva, no pico, até o topo da curva 5 minutos após o pico.

Propriedades de extensão da massa: determinadas no equipamento alveógrafo "Chopin", de acordo com o método da AACC n. ${ }^{\circ} 54-30$ A (AACC, 1995). Os parâmetros básicos das curvas alveográficas estudados foram os seguintes: pressão máxima $(\mathrm{P})$; abcissa média de ruptura (L); índice de configuração da curva $(\mathrm{P} / \mathrm{L})$; energia de deformação da massa (W) ou trabalho mecânico necessário para expandir a bolha até sua ruptura, expressa em $10^{-4}$ Joules.

Os genótipos estudados foram avaliados em soluções nutritivas contendo $0,2,4,6,8$ e $10 \mathrm{mg} . \mathrm{L}^{-1} \mathrm{de} \mathrm{Al}$, conforme Moore et al. (1976); Camargo e Oliveira (1981) e CAMARGO et al. (1987). 


\section{RESULTADOS E DISCUSSÃO}

No resumo das análises da variância para rendimento de grãos, apresentado no quadro 1, ocorreram efeitos significativos para anos, genótipos e interação anos $\mathrm{x}$ genótipos, nas quatro regiões. A significância da interação entre genótipos e anos para todos os locais foi indicativo para se proceder ao estudo da estabilidade fenotípica, pois os genótipos apresentaram respostas às variações regionais, sugerindo a existência de genótipos ou grupos de genótipos de adaptação específica ou ampla a todos os locais. $\mathrm{O}$ coeficiente de variação para ano e para genótipos refletiu a precisão dos experimentos, dentro dos valores aceitáveis para a espécie.

Em Tatuí (Quadro 1), na comparação das médias de rendimento, destacaram-se, pelo teste de Duncan ao nível de 5\%, os genótipos IAC 351, IAC 335, IAC 289 e Mochis; em Votuporanga, IAC 289, TUI“S” e SERI*3/BUC; em Ribeirão Preto e Mococa, o genótipo IAC 370.

Com base na interação genótipo $x$ ambiente, foram efetuadas as análises de adaptabilidade e estabilidade

Quadro 1. Produtividade média $\left(\mathrm{kg} \cdot \mathrm{ha}^{-1}\right)$ de grãos dos genótipos de trigo avaliados individualmente e em conjunto, em condição de irrigação por aspersão, em Tatuí (Zona D), Votuporanga (Zona F), Ribeirão Preto (Zona G) e Mococa (Zona H), Estado de São Paulo, de 1995 a 1998

\begin{tabular}{|c|c|c|c|c|c|}
\hline Genótipos & Tatuí & Votuporanga & Ribeirão Preto & Mococa & Médias \\
\hline & \multicolumn{5}{|c|}{$\longrightarrow \mathrm{kg} \cdot \mathrm{ha}^{-1}$} \\
\hline 1. Anahuac & $2.959 \mathrm{i}$ & $2.654 \mathrm{~g}$ & $3.888 \mathrm{~h}$ & $2.932 \mathrm{~h}$ & $3.108 \mathrm{e}$ \\
\hline 2. IAC 24 & $3.799 \mathrm{~b}-\mathrm{d}$ & $2.912 \mathrm{~d}-\mathrm{g}$ & $4.455 \mathrm{~d}-\mathrm{g}$ & $3.420 \mathrm{~d}-\mathrm{g}$ & $3.646 \mathrm{~b}-\mathrm{d}$ \\
\hline 3. IAC 60 & $3.755 \mathrm{~b}-\mathrm{d}$ & $2.990 \mathrm{~b}-\mathrm{f}$ & $4.368 \mathrm{e}-\mathrm{g}$ & $3.307 \mathrm{e}-\mathrm{g}$ & $3.605 \mathrm{c}-\mathrm{d}$ \\
\hline 4. IAC 286 & $3.740 \mathrm{~b}-\mathrm{d}$ & 3.141 a-e & $4.858 \mathrm{~b}-\mathrm{d}$ & $3.486 \mathrm{c}-\mathrm{f}$ & 3.806 a-d \\
\hline 5. IAC 287 & $3.388 \mathrm{e}-\mathrm{g}$ & $2.958 \mathrm{c}-\mathrm{f}$ & $4.326 \mathrm{f}-\mathrm{g}$ & $3.278 \mathrm{e}-\mathrm{g}$ & $3.487 \mathrm{~d}$ \\
\hline 6. IAC 289 & $3.869 \mathrm{a}-\mathrm{c}$ & $3.414 \mathrm{a}$ & $5.024 \mathrm{~b}-\mathrm{c}$ & $3.830 \mathrm{~b}$ & $4.034 \mathrm{a}-\mathrm{b}$ \\
\hline 7. JCAM/ /EMU "S" /YACO"S" & $3.607 \mathrm{c}-\mathrm{e}$ & $3.247 \mathrm{a}-\mathrm{c}$ & $4.488 \mathrm{~d}-\mathrm{g}$ & $3.162 \mathrm{f}-\mathrm{h}$ & $3.626 \mathrm{c}-\mathrm{d}$ \\
\hline 8. IAC 315 & $3.522 \mathrm{~d}-\mathrm{f}$ & $3.017 \mathrm{~b}-\mathrm{f}$ & $4.284 \mathrm{f}-\mathrm{g}$ & $3.244 \mathrm{f}-\mathrm{h}$ & $3.516 \mathrm{~d}$ \\
\hline 9. IAC 334 & $3.187 \mathrm{~g}-\mathrm{i}$ & $3.178 \mathrm{a}-\mathrm{d}$ & $4.978 \mathrm{~b}-\mathrm{c}$ & $3.413 \mathrm{~d}-\mathrm{g}$ & $3.689 \mathrm{~b}-\mathrm{d}$ \\
\hline 10. IAC 335 & $3.970 \mathrm{a}-\mathrm{b}$ & $2.751 \mathrm{f}-\mathrm{g}$ & $4.177 \mathrm{~g}-\mathrm{h}$ & $3.158 \mathrm{f}-\mathrm{h}$ & $3.514 \mathrm{~d}$ \\
\hline 11. IAC 352 & $3.548 \mathrm{c}-\mathrm{e}$ & $3.280 \mathrm{a}-\mathrm{b}$ & $4.865 \mathrm{~b}-\mathrm{d}$ & $3.372 \mathrm{~d}-\mathrm{g}$ & $3.743 \mathrm{a}-\mathrm{d}$ \\
\hline 12. Mochis & $3.868 \mathrm{a}-\mathrm{c}$ & 3.119 a-e & $5.168 \mathrm{~b}$ & $3.610 \mathrm{~b}-\mathrm{e}$ & $3.941 \mathrm{a}-\mathrm{c}$ \\
\hline 13. CAL/CHKW//VEE"S" & $3.221 \mathrm{f}-\mathrm{i}$ & $2.844 \mathrm{e}-\mathrm{g}$ & $4.469 \mathrm{~d}-\mathrm{g}$ & $3.171 \mathrm{f}-\mathrm{h}$ & 3.426 d-e \\
\hline 14. PFAU & $3.360 \mathrm{e}-\mathrm{h}$ & $3.295 a-b$ & $4.661 \mathrm{c}-\mathrm{f}$ & $3.129 \mathrm{~g}-\mathrm{h}$ & $3.611 \mathrm{c}-\mathrm{d}$ \\
\hline 15. TUI "S" & 3.635 c-e & $3.364 \mathrm{a}$ & $4.820 \mathrm{~b}-\mathrm{d}$ & $3.406 \mathrm{~d}-\mathrm{g}$ & $3.806 \mathrm{a}-\mathrm{d}$ \\
\hline 16. IAC 339 & $3.060 \mathrm{~h}-\mathrm{i}$ & $3.203 \mathrm{a}-\mathrm{d}$ & $4.760 \mathrm{~b}-\mathrm{e}$ & $3.324 \mathrm{e}-\mathrm{g}$ & $3.586 \mathrm{c}-\mathrm{d}$ \\
\hline 17. BAU/SERI & $3.570 \mathrm{c}-\mathrm{e}$ & $3.281 \mathrm{a}-\mathrm{b}$ & $4.449 \mathrm{~d}-\mathrm{g}$ & $3.685 \mathrm{~b}-\mathrm{d}$ & 3.746 a-d \\
\hline 18. SERI*3/BUC & $3.659 \mathrm{~b}-\mathrm{e}$ & $3.409 \mathrm{a}$ & $4.792 \mathrm{~b}-\mathrm{d}$ & $3.435 \mathrm{~d}-\mathrm{g}$ & 3.823 a-d \\
\hline 19. IAC 370 & $3.482 \mathrm{~d}-\mathrm{g}$ & 3.101 a-e & $5.632 \mathrm{a}$ & $4.292 \mathrm{a}$ & $4.126 \mathrm{a}$ \\
\hline 20. IAC 351 & $4.158 \mathrm{a}$ & $2.916 \mathrm{~d}-\mathrm{g}$ & $4.844 \mathrm{~b}-\mathrm{d}$ & $3.784 \mathrm{~b}-\mathrm{c}$ & $3.925 \mathrm{a}-\mathrm{c}$ \\
\hline Médias & $3.568 \mathrm{~B}$ & $3.104 \mathrm{C}$ & $4.665 \mathrm{~A}$ & $3.422 \mathrm{~B}$ & 3.688 \\
\hline $\mathrm{F}$ (ano) & $503,05^{* *}$ & $140,06^{* *}$ & $103,29^{* *}$ & $46,67^{* *}$ & \\
\hline F (genótipo) & $9,22^{* *}$ & $5,07^{* *}$ & $8,95^{* *}$ & $7,92^{* *}$ & $3,66^{*}$ \\
\hline F (ano x genótipo) & $3,99 * *$ & $1,62^{* *}$ & $2,61^{* *}$ & $2,06^{* *}$ & \\
\hline $\mathrm{CV} \%$ - ano & 2,76 & 4,17 & 2,20 & 5,99 & \\
\hline CV\% - genótipo & 11,32 & 12,46 & 11,24 & 12,66 & 6,55 \\
\hline
\end{tabular}

Médias para comparação da produtividade de grãos entre cultivares por local e na média de locais em letras minúsculas; médias para comparação entre locais em letras maiúsculas. Médias seguidas por letras distintas diferem, entre si, pelo teste de Duncan ao nível de $5 \%$. ${ }^{* * *}$ : significativo ao nível de $5 \%$ e $1 \%$, respectivamente, pelo teste $\mathrm{F}$. 
Influência do ambiente no rendimento e qualidade de grãos de trigo com irrigação por aspersão

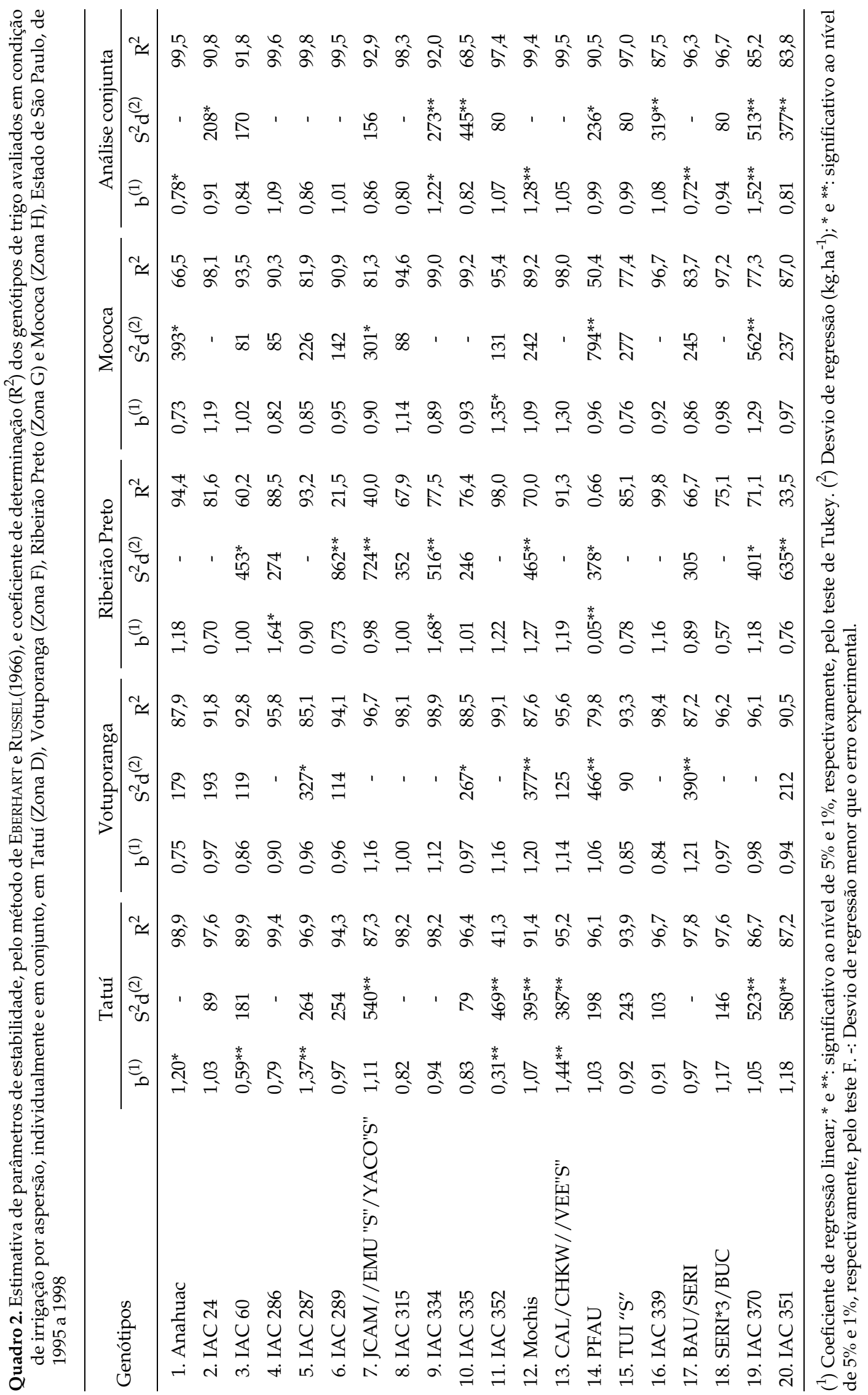


(Quadro 2), que possibilitaram prever o comportamento dos genótipos em face das variações ambientais.

Os genótipos Anahuac, IAC 287 e CAL/CHKW / / $\mathrm{VEE}^{\text {" }} \mathrm{S}$ " apresentaram-se responsivos à melhoria do ambiente, em Tatuí; os genótipos IAC 286 e IAC 334, em Ribeirão Preto; o IAC 352, em Mococa. Como responsivos às condições adversas, destacaram-se os genótipos IAC 60 e IAC 352, em Tatuí, e o PFAU, em Ribeirão Preto.

Geralmente, considera-se como genótipo responsivo aquele que apresenta alta capacidade produtiva em ambientes favoráveis e baixa resposta em desfavoráveis. No estudo em questão, o genótipo IAC 370 pode ser considerado como o mais responsivo, quando analisados todos os experimentos em conjunto.

Dependendo das condições climáticas adversas, aliadas à suscetibilidade dos genótipos, a cultura do trigo poder ter seus rendimentos reduzidos pelo ata- que de doenças causadas por fungos, como as ferrugens, manchas foliares e o oídio.

A ferrugem-da-folha, de abrangência e ocorrência mais generalizada entre as diversas doenças do trigo (BARCELlOS, 1982), foi mais relevante em Tatuí, seguida de Ribeirão Preto e Mococa (Quadro 3). Em Votuporanga, não se verificou a presença da doença. Em Tatuí, os genótipos IAC 287, Mochis, IAC 339 e IAC 370 apresentaram os menores índices de suscetibilidade ao agente causal da doença.

A ocorrência do oídio, variável de acordo com as condições climáticas do ano, depende de condições favoráveis para o seu desenvolvimento; em sistema de irrigação por aspersão, tem-se mostrado presente em todas as zonas tritícolas paulistas, conforme observações de FeLICIO et al. (1996). Os genótipos que apresentam incidência nas folhas superior a $25 \%$ estão no limiar de ação para o início do controle químico (CAmpinas, 1999). Em Tatuí, região que se mostrou mais favorável ao fungo, destacaram-se Anahuac,

Quadro 3. Avaliação da ocorrência de ferrugem-da-folha (FF), manchas foliares $(\mathrm{MF})$ e oídio $(\mathrm{O})$, em porcentagem de área foliar infectada, nos genótipos de trigo avaliados em condição de irrigação por aspersão, em Tatuí (Zona D), Votuporanga (Zona F), Ribeirão Preto (Zona G) e Mococa (Zona H), Estado de São Paulo, de 1995 a 1998

\begin{tabular}{|c|c|c|c|c|c|c|c|c|c|c|c|c|}
\hline \multirow{2}{*}{ Genótipos } & \multicolumn{3}{|c|}{ Tatuí } & \multicolumn{3}{|c|}{ Votuporanga } & \multicolumn{3}{|c|}{ Ribeirão Preto } & \multicolumn{3}{|c|}{ Mococa } \\
\hline & $\mathrm{FF}$ & MF & $\mathrm{O}$ & $\mathrm{FF}$ & MF & $\mathrm{O}$ & FF & MF & $\mathrm{O}$ & FF & MF & $\mathrm{O}$ \\
\hline 1. Anahuac & 45 & 20 & 30 & - & 5 & - & 5 & 10 & 25 & 10 & 5 & - \\
\hline 2. IAC 24 & 40 & 30 & 15 & - & 5 & - & 5 & 10 & 5 & 5 & 5 & - \\
\hline 3. IAC 60 & 25 & 25 & 10 & - & 5 & - & 5 & 10 & $\mathrm{t}$ & $\mathrm{t}$ & 5 & - \\
\hline 4. IAC 286 & 30 & 30 & 25 & - & 5 & - & 5 & 10 & $\mathrm{t}$ & 0 & 5 & - \\
\hline 5. IAC 287 & 5 & 25 & 35 & - & 5 & - & 5 & 10 & 10 & 0 & 5 & - \\
\hline 6. IAC 289 & 25 & 25 & 10 & - & 5 & - & 15 & 10 & 0 & 0 & 5 & - \\
\hline 7. JCAM//EMU "S"/YACO"S" & 20 & 30 & 5 & - & 5 & - & $\mathrm{t}$ & 10 & 5 & 5 & 5 & - \\
\hline 8. IAC 315 & 35 & 30 & 10 & - & 5 & - & 5 & 10 & $\mathrm{t}$ & 0 & 5 & - \\
\hline 9. IAC 334 & 20 & 35 & 20 & - & 5 & - & $\mathrm{t}$ & 10 & 10 & 5 & 5 & - \\
\hline 10. IAC 335 & 20 & 20 & 10 & - & 5 & - & 0 & 10 & 5 & 0 & 5 & - \\
\hline 11. IAC 352 & 10 & 35 & 10 & - & 5 & - & 5 & 10 & $\mathrm{t}$ & 0 & 5 & - \\
\hline 12. Mochis & 5 & 30 & 15 & - & 5 & - & 5 & 10 & $\mathrm{t}$ & 0 & 5 & - \\
\hline 13. CAL/CHKW//VEE"S" & 15 & 30 & 30 & - & 5 & - & $\mathrm{t}$ & 10 & 10 & 0 & 5 & - \\
\hline 14. PFAU & 30 & 30 & 5 & - & 5 & - & 10 & 10 & $\mathrm{t}$ & $\mathrm{t}$ & 5 & - \\
\hline 15. TUI "S" & 20 & 20 & 25 & - & 5 & - & 5 & 10 & $\mathrm{t}$ & 5 & 5 & - \\
\hline 16. IAC 339 & 5 & 31 & 15 & - & 5 & - & 5 & 10 & 5 & 0 & 5 & - \\
\hline 17. BAU/SERI & 20 & 25 & 15 & - & 5 & - & $\mathrm{t}$ & 10 & 5 & 5 & 5 & - \\
\hline 18. SERI*3/BUC & 10 & 30 & 15 & - & 5 & - & $\mathrm{t}$ & 10 & 0 & 0 & 5 & - \\
\hline 19. IAC 370 & 5 & 25 & 35 & - & 5 & - & $\mathrm{t}$ & 10 & 10 & 5 & 5 & - \\
\hline 20. IAC 351 & 30 & 25 & 20 & - & 5 & - & $\mathrm{t}$ & 10 & 20 & 10 & 5 & - \\
\hline
\end{tabular}

-: Não houve ocorrência da doença. 
IAC 287, CAL/CHKW//VEE“S" e IAC 370. Em Ribeirão Preto, somente o Anahuac atingiu o índice de $25 \%$ de área foliar infectada pela doença (Quadro 3).

As manchas foliares causadas por Bipolaris sorokiniana (mancha-marrom) e Drechslera tritici repentis (mancha-bronzeada-da-folha) desenvolveram-se mais intensamente em Tatuí (Quadro 3), com porcentagem de área foliar infectada que varia de $20 \%$ a $35 \%$ entre os genótipos. Nas demais regiões, a incidência não foi relevante a ponto de causar danos ao rendimento do genótipo infectado.

Tanto o genótipo como os fatores ambientais e os associados ao cultivo influenciam a qualidade industrial de um trigo. Como as características tecnológicas do grão da maioria dos 20 genótipos em estudo já são conhecidas, fez-se necessário verificar as variações dessas características entre os anos (safras). Nos quadros 4 e 5 são apresentados os resultados das análises dos 20 genótipos de Triticum aestivum L. semeados em Ribeirão Preto, nos anos de 1996-97.

Os resultados mostram baixa variação entre os anos para $\mathrm{PH}$ (peso hectolítrico). Com relação à extração experimental de farinha, os resultados estão de acordo com o padrão para moinhos experimentais, acima de 70\%, exceto para os genótipos IAC 60, IAC 287 e CAL/CHKW//VEE"S", cujos índices foram inferiores nos anos analisados.

A quantidade de proteína do grão de trigo pode variar entre $9-17 \%$, dependendo dos fatores genéticos, ambientais e daqueles associados ao cultivo (PeÑa et al., 1998); a quantidade e a qualidade da proteína determinam a força do glúten. Verificou-se, nas amostras avaliadas, que a quantidade de proteína da farinha está acima do limite mínimo de $9 \%$, e que o genótipo IAC 24 e o IAC 351 apresentaram, nos dois anos, níveis acima de $14 \%$.

Quadro 4. Peso hectolítrico (PH), extração experimental de farinha (Ext), proteína da farinha (PRTF), número de queda (NQ) e características farinográficas (tempo de desenvolvimento da massa - TDM; estabilidade - EST e índice de tolerância à mistura ITM ) e alveográficas (P/L e W) dos genótipos de Triticum aestivum L., avaliados em condição de irrigação por aspersão, em Ribeirão Preto (Zona G), Estado de São Paulo, em 1996

\begin{tabular}{|c|c|c|c|c|c|c|c|c|c|}
\hline \multirow{2}{*}{ Genótipos } & \multirow{2}{*}{$\mathrm{PH}$} & \multirow{2}{*}{ Ext } & \multirow{2}{*}{ PRTF } & \multirow{2}{*}{ NQ } & \multicolumn{3}{|c|}{ Farinografia } & \multicolumn{2}{|c|}{ Alveografia } \\
\hline & & & & & TDM & EST & $\operatorname{ITM}^{(1)}$ & $\mathrm{P} / \mathrm{L}^{(2)}$ & $\mathrm{W}^{(3)}$ \\
\hline & 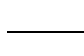 & - \% - & - & $\mathrm{s}$ & $\min$ & $\min$ & & & $10^{-4} \mathrm{~J}$ \\
\hline 1. Anahuac & 80,1 & 73,53 & 13,7 & 393 & 9,40 & 18,80 & 15 & 1,53 & 358 \\
\hline 2. IAC 24 & 77,2 & 70,45 & 16,4 & 419 & 8,70 & 9,70 & 35 & 0,89 & 395 \\
\hline 3. IAC 60 & 75,2 & 67,04 & 13,6 & 359 & 5,30 & 6,40 & 60 & 0,43 & 191 \\
\hline 4. IAC 286 & 79,9 & 70,96 & 13,3 & 228 & 5,80 & 7,00 & 60 & 0,73 & 238 \\
\hline 5. IAC 287 & 77,2 & 64,83 & 12,4 & 302 & 4,70 & 16,30 & 25 & 0,76 & 199 \\
\hline 6. IAC 289 & 76,8 & 74,09 & 15,0 & 222 & 6,20 & 7,00 & 50 & 0,67 & 250 \\
\hline 7. JCAM//EMU"S"/YACO"S" & 78,3 & 76,34 & 13,4 & 174 & 4,50 & 6,50 & 60 & 1,45 & 252 \\
\hline 8. IAC 315 & 78,3 & 72,72 & 15,6 & 321 & 7,30 & 8,40 & 40 & 1,29 & 345 \\
\hline 9. IAC 334 & 81,2 & 74,58 & 12,7 & 255 & 5,80 & 9,60 & 40 & 1,53 & 304 \\
\hline 10. IAC 335 & 78,5 & 73,22 & 15,0 & 322 & 6,00 & 6,30 & 60 & 0,49 & 222 \\
\hline 11. IAC 352 & 78,6 & 72,23 & 14,6 & 410 & 5,20 & 4,60 & 60 & 0,78 & 208 \\
\hline 12. Mochis & 79,7 & 72,47 & 15,1 & 411 & 5,70 & 4,80 & 35 & 1,55 & 211 \\
\hline 13. CAL/CHKW//VEE"S" & 80,3 & 67,18 & 13,2 & 350 & 5,50 & 5,60 & 45 & 1,17 & 179 \\
\hline 14. PFAU & 78,6 & 74,50 & 14,1 & 295 & 7,10 & 10,90 & 30 & 1,39 & 306 \\
\hline 15. TUI "S" & 81,7 & 75,10 & 13,3 & 417 & 9,10 & 13,80 & 25 & 1,51 & 313 \\
\hline 16. IAC 339 & 82,6 & 76,31 & 13,3 & 249 & 8,40 & 12,10 & 35 & 2,16 & 305 \\
\hline 17. BAU/SERI & 76,8 & 73,74 & 12,6 & 237 & 8,00 & 9,40 & 60 & 1,49 & 256 \\
\hline 18. SERI*3/BUC & 76,8 & 72,20 & 14,1 & 203 & 4,40 & 4,10 & 80 & 1,26 & 160 \\
\hline 19. IAC 370 & 77,7 & 72,18 & 13,4 & 314 & 6,80 & 8,60 & 60 & 1,33 & 255 \\
\hline 20. IAC 351 & 78,6 & 71,74 & 15,5 & 409 & 7,80 & 11,10 & 40 & 0,78 & 306 \\
\hline
\end{tabular}

$\left({ }^{1}\right)$ Unidades farinográficas. $\left({ }^{2}\right)$ Índice de configuração da curva. $\left({ }^{3}\right)$ Força do glúten. 
Quadro 5. Peso hectolítrico (PH), extração experimental de farinha (Ext), proteína da farinha (PRTF), número de queda (NQ) e características farinográficas (tempo de desenvolvimento da massa - TDM, estabilidade - EST e índice de tolerância à mistura ITM ) e alveográficas (P/L e W) dos genótipos de Triticum aestivum L., avaliados em condição de irrigação por aspersão, em Ribeirão Preto (Zona G), Estado de São Paulo, em 1997

\begin{tabular}{|c|c|c|c|c|c|c|c|c|c|}
\hline \multirow{2}{*}{ Genótipos } & \multirow{2}{*}{$\mathrm{PH}$} & \multirow{2}{*}{ Ext } & \multirow{2}{*}{ PRTF } & \multirow{2}{*}{ NQ } & \multicolumn{3}{|c|}{ Farinografia } & \multicolumn{2}{|c|}{ Alveografia } \\
\hline & & & & & TDM & EST & $\operatorname{ITM}^{(1)}$ & $\mathrm{P} / \mathrm{L}^{(2)}$ & $\mathrm{W}^{(3)}$ \\
\hline & 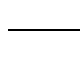 & $-\%$ & - & $\mathrm{s}$ & $\min$ & $\min$ & & & $10^{-4} \mathrm{~J}$ \\
\hline 1. Anahuac & 81,2 & 74,68 & 10,6 & 434 & 6,2 & 11,8 & 25 & 2,18 & 249 \\
\hline 2. IAC 24 & 79,7 & 71,17 & 14,4 & 526 & 6,6 & 8,8 & 25 & 1,04 & 351 \\
\hline 3. IAC 60 & 79,7 & 69,66 & 12,0 & 386 & 4,0 & 4,4 & 70 & 0,40 & 147 \\
\hline 4. IAC 286 & 81,5 & 72,73 & 13,3 & 448 & 5,0 & 4,5 & 55 & 0,79 & 209 \\
\hline 5. IAC 287 & 79,1 & 67,11 & 11,2 & 389 & 4,5 & 6,5 & 50 & 0,69 & 199 \\
\hline 6. IAC 289 & 80,6 & 75,60 & 12,5 & 423 & 7,0 & 7,2 & 35 & 1,09 & 222 \\
\hline 7. JCAM//EMU"S"/YACO"S" & 81,0 & 76,06 & 11,5 & 386 & 4,9 & 9,9 & 20 & 1,93 & 284 \\
\hline 8. IAC 315 & 79,4 & 71,66 & 13,2 & 484 & 6,5 & 6,9 & 35 & 1,16 & 240 \\
\hline 9. IAC 334 & 83,5 & 75,06 & 12,2 & 469 & 7,3 & 7,1 & 50 & 1,72 & 272 \\
\hline 10. IAC 335 & 79,9 & 72,52 & 13,2 & 389 & 4,9 & 4,0 & 50 & 0,71 & 195 \\
\hline 11. IAC 352 & 81,7 & 73,24 & 13,1 & 460 & 3,8 & 3,0 & 55 & 0,75 & 169 \\
\hline 12. Mochis & 81,7 & 73,71 & 13,7 & 462 & 3,8 & 1,5 & 80 & 2,90 & 163 \\
\hline 13. CAL/CHKW//VEE & 81,2 & 68,06 & 13,1 & 384 & 3,8 & 4,0 & 55 & 1,44 & 146 \\
\hline 14. PFAU & 82,9 & 76,33 & 13,2 & 382 & 7,3 & 8,1 & 30 & 2,00 & 341 \\
\hline 15. TUI "S" & 82,6 & 76,04 & 12,6 & 494 & 9,3 & 10,0 & 40 & 1,36 & 275 \\
\hline 16. IAC 339 & 83,3 & 76,35 & 13,5 & 458 & 9,1 & 9,6 & 30 & 0,81 & 287 \\
\hline 17. BAU/SERI & 79,9 & 73,82 & 12,4 & 352 & 6,0 & 7,4 & 35 & 0,88 & 211 \\
\hline 18. SERI*3/BUC & 81,0 & 73,91 & 12,2 & 443 & 5,3 & 5,6 & 45 & 0,84 & 140 \\
\hline 19. IAC 370 & 82,1 & 74,99 & 12,2 & 462 & 8,1 & 14,0 & 20 & 1,06 & 265 \\
\hline 20. IAC 351 & 77,4 & 73,64 & 14,2 & 496 & 6,7 & 7,6 & 30 & 0,47 & 246 \\
\hline
\end{tabular}

$\left({ }^{1}\right)$ Unidades farinográficas. $\left({ }^{2}\right)$ Índice de configuração da curva. $\left({ }^{3}\right)$ Força do glúten.

Os dados do número de queda (NQ) mostraram que não ocorreu germinação dos grãos na espiga. Esse fato indica que as variações climáticas das regiões não foram significativas para alterar a atividade enzimática dos grãos, exceto para o genótipo JCAM// EMU"S"/YACO"S", que apresentou número de queda de 174 segundos em 1996, valor abaixo do padrão para trigo tipo "pão" (BRASIL, 1999).

Nos dados obtidos no farinógrafo, para as 20 amostras, na análise dos parâmetros tempo de desenvolvimento da massa, estabilidade e índice de tolerância à mistura, de acordo com o que estabeleceram PeÑA et al. (1998), os genótipos Anahuac, IAC 24, IAC 287, IAC 289, IAC 334, PFAU, TUI“S”, IAC 339, IAC 370 e IAC 351 apresentaram características de farinha com bom potencial para panificação. Os demais genótipos são mais adequados para utilização na indústria de biscoitos, confeitaria ou massas frescas. Nenhum genótipo apresentou qualidade tecnológica excessivamente baixa.

O genótipo IAC 24 é classificado como trigo "melhorador" por Germani e Carvalho (1998) e, igualmente, pela Instrução Normativa n. ${ }^{\circ} 1$ do Ministério da Agricultura e do Abastecimento (BRASIL, 1999), segundo os quais a classe "melhorador" caracterizase por força do glúten com o mínimo de $300\left(\times 10^{-4} \mathrm{~J}\right)$. Os dados de 1997 permitem alocar, na mesma classe, o genótipo PFAU. A análise das amostras de 1996 indicou, além destes genótipos, Anahuac, TUI"S", IAC 339 e IAC 351, como trigos tipo "melhorador". Classificaram-se como tipo "pão", na média dos dois anos, Anahuac, IAC 286, IAC 287, IAC 289, JCAM/ /EMU“S" /YACO"S", IAC 315, IAC 334, IAC 335, TUI“S”, IAC 339, BAU/SERI, IAC 370 e IAC 351 e, na classe "outros usos", os demais genótipos.

Os resultados das análises para qualidade tecnológica dos grãos, das safras de 1996 e 1997 reali- 
Quadro 6. Comprimento médio das raízes dos 20 genótipos de trigo, avaliado após 72 horas de crescimento em solução normal, que se seguiu a um crescimento em solução-tratamento contendo seis diferentes concentrações de alumínio

\begin{tabular}{|c|c|c|c|c|c|c|}
\hline \multirow{2}{*}{ Genótipos } & \multicolumn{6}{|c|}{ Concentrações de alumínio (mg. $\mathrm{L}^{-1}$ ) } \\
\hline & 0 & 2 & 4 & 6 & 8 & 10 \\
\hline & & & & 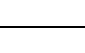 & 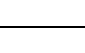 & 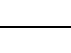 \\
\hline 1. Anahuac & 77,2 & 0,0 & 0,0 & 0,0 & 0,0 & 0,0 \\
\hline 2. IAC 24 & 76,4 & 59,5 & 55,7 & 51,8 & 37,5 & 23,5 \\
\hline 3. IAC 60 & 63,4 & 73,2 & 58,7 & 33,4 & 16,3 & 7,3 \\
\hline 4. IAC 286 & 68,7 & 69,2 & 51,3 & 50,7 & 39,8 & 23,9 \\
\hline 5. IAC 287 & 84,6 & 0,0 & 0,0 & 0,0 & 0,0 & 0,0 \\
\hline 6.IAC 289 & 68,6 & 50,3 & 6,4 & 3,3 & 0,0 & 0,0 \\
\hline 7. JCAM//EMU “S" / YACO “S” & 79,7 & 0,0 & 0,0 & 0,0 & 0,0 & 0,0 \\
\hline 8. IAC 315 & 58,7 & 55,0 & 49,3 & 44,0 & 30,4 & 28,3 \\
\hline 9. IAC 334 & 61,7 & 69,0 & 51,9 & 49,9 & 36,9 & 31,7 \\
\hline 10. IAC 335 & 90,3 & 75,2 & 52,2 & 45,3 & 39,5 & 31,2 \\
\hline 11. IAC 352 & 96,6 & 61,9 & 52,3 & 43,7 & 25,1 & 5,8 \\
\hline 12. Mochis & 76,1 & 58,3 & 53,5 & 39,4 & 26,1 & 7,4 \\
\hline 13. CAL/CHKW//VEE “S” & 80,2 & 42,5 & 0,0 & 0,0 & 0,0 & 0,0 \\
\hline 14. PFAU & 91,2 & 0,0 & 0,0 & 0,0 & 0,0 & 0,0 \\
\hline 15. TUI “S” & 88,9 & 11,6 & 0,0 & 0,0 & 0,0 & 0,0 \\
\hline 16. IAC 339 & 81,5 & 0,0 & 0,0 & 0,0 & 0,0 & 0,0 \\
\hline 17. BAU/SERI & 82,1 & 39,6 & 6,1 & 0,0 & 0,0 & 0,0 \\
\hline 18. SERI *3/BUC & 86,1 & 56,2 & 15,3 & 0,7 & 0,0 & 0,0 \\
\hline 19. IAC 370 & 109,0 & 55,6 & 2,0 & 0,0 & 0,0 & 0,0 \\
\hline 20. IAC 351 & 109,0 & 92,0 & 58,0 & 34,6 & 14,3 & 5,2 \\
\hline
\end{tabular}

zadas com as sementes provenientes de Ribeirão Preto, apresentaram melhor qualidade tecnológica para a última. Essas amostras apresentaram número de queda mais alto; representa, portanto, menor atividade enzimática nos grãos. Nos resultados de farinografia e alveografia, algumas exceções ocorreram, como no genótipo Anahuac, que apresentou melhor desempenho para força do glúten em 1996 e quando comparado a 1997.

O comprimento médio das raízes dos genótipos estudados, medidas após 72 horas de crescimento em solução nutritiva, que se seguiu a um crescimento em solução-tratamento contendo seis diferentes concentrações de alumínio, encontra-se no quadro 6 . Verificou-se que mediante o aumento da concentração de alumínio ocorreu uma diminuição no crescimento das raízes, e que a intensidade dessa diminuição corresponde ao grau de tolerância de cada genótipo ao alumínio. De acordo com CAMARGo e Felicio (1984), os seguintes genótipos podem ser classificados como suscetíveis: Anahuac, IAC 287, JCAM//EMU"S" / YACO"S", PFAU e IAC 339; como moderadamente suscetíveis, CAL/CHKW//VEE"S", TUI“S", BAU/ SERI e IAC 370; como moderadamente tolerantes, IAC 289 e SERI*3/BUC e, como tolerantes, IAC 24, IAC 60, IAC 286, IAC 315, IAC 334, IAC 335, IAC 352, Mochis e IAC 351.

\section{CONCLUSÕES}

1. As diferenças entre as regiões contribuíram para influenciar o rendimento de grãos entre os locais estudados.

2. Os genótipos Anahuac, IAC 287, JCAM// EMU“S" /YACO"S", PFAU e IAC 339 foram os mais sensíveis ao alumínio tóxico.

3. IAC 370 apresentou alta produtividade, baixa resposta nos ambientes desfavoráveis e foi responsivo nos ambientes favoráveis.

4. A ocorrência de ferrugem-da-folha foi mais intensa em Tatuí, onde os genótipos IAC 287, Mochis, IAC 339 e IAC 370 foram os mais resistentes ao agente causal da doença. 
5. A intensidade da ocorrência de oídio variou entre as regiões, apesar da irrigação; Tatuí foi o local que apresentou maior incidência. Destacaram-se como os mais suscetíveis (30-35\% de área foliar infectada) os genótipos Anahuac, IAC 287, CAL/ CHKW/ / VEE"S" e IAC 370.

6. Os genótipos Anahuac, IAC 286, IAC 287, IAC 289, JCAM//EMU “S" /YACO "S", IAC 315, IAC 334, IAC 335, TUI"S", IAC 339, BAU/SERI, IAC 370 e o IAC 351, pelas análises conjuntas (1996-97) dos dados obtidos no farinógrafo e alveógrafo, apresentaram características que os classificam como trigo tipo "pão", enquanto o IAC 24 e o PFAU, como trigo tipo "melhorador".

\section{AGRADECIMENTOS}

Aos técnicos de apoio José Norberto da Silva, Carlos Aparecido Fernandes, José Roberto Cassanelli Júnior, Edvaldo Novelli Gomes e Sérgio José Coradello pela colaboração no desenvolvimento dos experimentos.

\section{REFERÊNCIAS BIBLIOGRÁFICAS}

AACC. AMERICAN ASSOCIATION OF CEREAL CHEMISTS. Approved methods of AACC. 9.ed. St. Paul: AACC, 1995.

AOAC. ASSOCIATION OF OFFICIAL ANALYTICAL CHEMISTS. Official methods of analysis of AOAC. 16.ed. 3.rev. Gaitherburg: AOAC, 1997.

AZEVEDO, A.J.;SILVA, E.M. da; RESENDE, M.; GUERRA, A.F. Aspectos sobre o manejo da irrigação por aspersão para o cerrado. Brasília: EMBRAPA-DID, 1983. 53p. (EMBRAPA-CPAC. Circular Técnica, 16)

BARCELLOS, A.L. A ferrugem-do-trigo no Brasil. In: FUNDAÇÃO CARGILL. Trigo no Brasil. Campinas, 1982. v.2, p.377-410.

BRASIL. Instruções Normativas n. ${ }^{\circ}$ 1, de 27/1/99. Ministério da Agricultura e do Abastecimento. Diário Oficial da União, Brasília, seção 1. p.132, 29/1/99.

CAMARGO, C.E. de O.; FELICIO, J.C. Tolerância de cultivares de trigo, triticale e centeio em diferentes níveis de alumínio em solução nutritiva. Bragantia, Campinas, v.43, n.1, p.9-16, 1984.

CAMARGO, C.E. de O.; FELICIO, J.C.; ROCHA JUNIOR, L.S. Trigo: tolerância ao alumínio em solução nutritiva. Bragantia, Campinas, v.46, n.2, p.183-190, 1987.

CAMARGO, C.E. de O.; OLIVEIRA, O.F. Tolerância de cultivares de trigo a diferentes níveis de alumínio em solução nutritiva e no solo. Bragantia, Campinas, v.40, n.1, p.21-31, 1981.

CAMPINAS, INSTITUTO AGRONÔMICO. Recomendações técnicas de trigo para 1999. 2.ed.atual. Campinas, 1999. 100p. (Boletim Técnico, 167)
EBERHART, S.A.; RUSSELL,W.A. Stability parameters for comparing varieties. Crop Science, Madison, v.6, p.3640, 1966.

FELICIO, J.C.; CAMARGO, C.E. de O.; MAGNO, C.P.R. dos S.; GALLO, P.B.; PEREIRA, J.C.V.N.A.; PETTINELLI JUNIOR, A. Avaliação agronômica e de qualidade tecnológica de genótipos de trigo com irrigação por aspersão no Estado de São Paulo. Bragantia, Campinas, v.55, n.1, p.147-156, 1996.

GERMANI, R.; CARVALHO, J.L.V. de. Perfil tecnológico de cultivares de trigo brasileiro. Rio de Janeiro: EMBRAPACTAA, 1998.72p. (EMBRAPA/CTAA-Documentos, 32)

GOEDERT, W.J.; LOBATO, E; WAGNER, E. Potencial agrícola da região dos cerrados brasileiros. Pesquisa Agropecuária Brasileira, Brasília, v.15, n.1, p.1-17, 1980.

GOMES, F.P. Curso de estatística experimental. 13.ed. São Paulo: Nobel, 1990. 430p.

LARGE, E.C. Growth stages in cereals. Illustration of the Feekes Scale. Plant Pathology, Oxford, v.3, p.128-129, 1954.

MALAVOLTA, E.; KLIEMANN, H.J. Desordens nutricionais no cerrado. Piracicaba: Potafos, 1985. 136p.

MOORE, D.P.; KRONSTAD, W.E.; METZGER, R.J. Screening wheat for aluminum tolerance. In: WORKSHOP ON PLANT ADAPTATIONS TO MINERAL STRESS IN PROBLEM SOILS, 1976, Beltsville, Maryland. Proceedings... Ithaca: Cornell University, 1976. p.287-295.

PEÑA, R.J.; ORTIZ-MONASTEIRO, J.I.; SAYRE, K.D. Estrategias para mejorar (o mantener) la calidad panadeira en trigo de alto potencial de rendimiento. In KOHLI, M.M. y MARTINO, D. (Eds.). Explorando altos rendimientos de trigo. La Estanzuela, Uruguay: CIMMYT-INIA, 1998. p.289-306.

RAIJ, B. van; CANTARELLA, H.; QUAGGIO, J.A.; FURLANI, A.M.C. Recomendações de adubação e calagem para o Estado de São Paulo. Campinas: Instituto Agronômico/Fundação IAC, 1996. 285p. (Boletim Técnico, 100)

REICHARDT, K. Processos de transferência no sistema soloplanta-atmosfera. 4.ed. Campinas: Fundação Cargill, 1985. 466p.

SCHARAMM, W.; FULCO, W.S.; SOARES, M.H.G.; ALMEIDA, A.M.P. Resistência de cultivares de trigo em experimentos ou cultivo no Rio Grande do Sul, às principais doenças fúngicas. Agronomia Sulriograndense, Porto Alegre, v.10, p.31-39, 1974.

SILVA, E.M.; LUCHIARI JÚNIOR, A.; GUERRA. A.F.; GOMIDE, R.L. Recomendações sobre manejo de irrigação em trigo para a região dos cerrados. In: REUNIÃO DA COMISSÃO NORTE-BRASILEIRA DE PESQUISA DE TRIGO, 10., 1984, Campinas. Ata... Brasília: EMBRAPA-CPAC, 1984. 60p.

WAL, P.C. Rendimientos y el cociente fototérmico: una relación inevitable? In: KOHLI, M.M. y MARTINO, D. (Eds.). Explorando altos rendimientos de trigo. La Estanzuela, Uruguay: CIMMYT-INIA, 1998. p.47-69. 